\title{
Kasuistiken
}

Hautarzt 2020 $\cdot 71: 805-808$

https://doi.org/10.1007/s00105-020-04654-8

Online publiziert: 21. Juli 2020

(c) Der/die Autor(en) 2020

Christiane S. Cussigh · Ferdinand Toberer · Alexander Enk · Holger A. Haenssle • Christine Fink

Klinik für Dermatologie, Venerologie und Allergologie, Universitätsklinikum Heidelberg (UKH), RuprechtKarls-Universität, Heidelberg, Deutschland

\section{Therapieansprechen auf intraläsionale Steroidinjektionen bei granulomatöser Entzündungsreaktion nach Tätowierungen}

\section{Anamnese}

Fall 1. Eine 60-jährige Patientin berichtete bei ihrer Vorstellung über seit 4 Monaten bestehende, schmerzlose, größenprogrediente Knoten an der Unterlippe nach Applikation eines PermanentMake-ups am Lippenrand 16 Monate zuvor (-Abb. 1a-c).

Fall 2. Die Vorstellung einer 56-jährigen Patientin erfolgte wegen seit 6 Monaten bestehender, juckender Knoten innerhalb roter Areale eines Tattoos am linken Unterschenkel, das vor 7 Jahren in Thailand gestochen wurde (• Abb. 1d-f).

Beide Patientinnen verneinten relevante Vorerkrankungen, eine Dauermedikation, Schmerzen oder Krankheitsgefühl.

\section{Klinik}

Fall 1. Bei der Inspektion des Gesichtes zeigten sich eine randbetonte weißliche Schuppung und gelbliche Krusten am Lippenrot der Ober- und Unterlippe (• Abb. 1a). Zudem imponierten infiltrierte Knoten und narbige Atrophien sowie eine rote, linienförmige Tätowierung entlang der Lippenkontur.

Fall 2. Am linken Unterschenkel distal lateral fanden sich multiple, derbe Knoten ausschließlich in den roten Arealen einer Tätowierung (• Abb. 1d).
Das übrige Integument inklusive der Mundschleimhaut war in beiden Fällen unauffällig.

\section{Diagnostik}

Bei Verdacht auf eine allergische Typ-IVReaktion auf Bestandteile des Tattoofarbstoffes sowie zum differenzialdiagnostischen Ausschluss einer granulomatösen Entzündungsreaktion infektiöser Genese wurde jeweils eine Probebiopsie entnommen.

\section{Histopathologischer und laborchemischer Befund}

Fall 1. Die Histologie zeigte unter einem hyperplastischen, spongiotisch aufgelockerten Epithel eine granulomatöse Entzündungsreaktion um exogenes rotes und schwarzes Pigment in der oberen Dermis sowie begleitend ein plasmazellreiches, lymphozytäres Infiltrat (- Abb. 2a, b).

Die Treponemen-, PAS(„periodic acid-Schiff reaction")- und Ziehl-Neelsen-Färbung ergaben keinen Anhalt auf pathogene Organismen. Eine aufgrund des Plasmazellreichtums durchgeführte Luesserologie und ein QuantiferonTest (Quantiferon-TB-Gold-Test, Qiagen $\mathrm{GmbH}$, Hilden, Deutschland) waren unauffällig. 


\section{Kasuistiken}
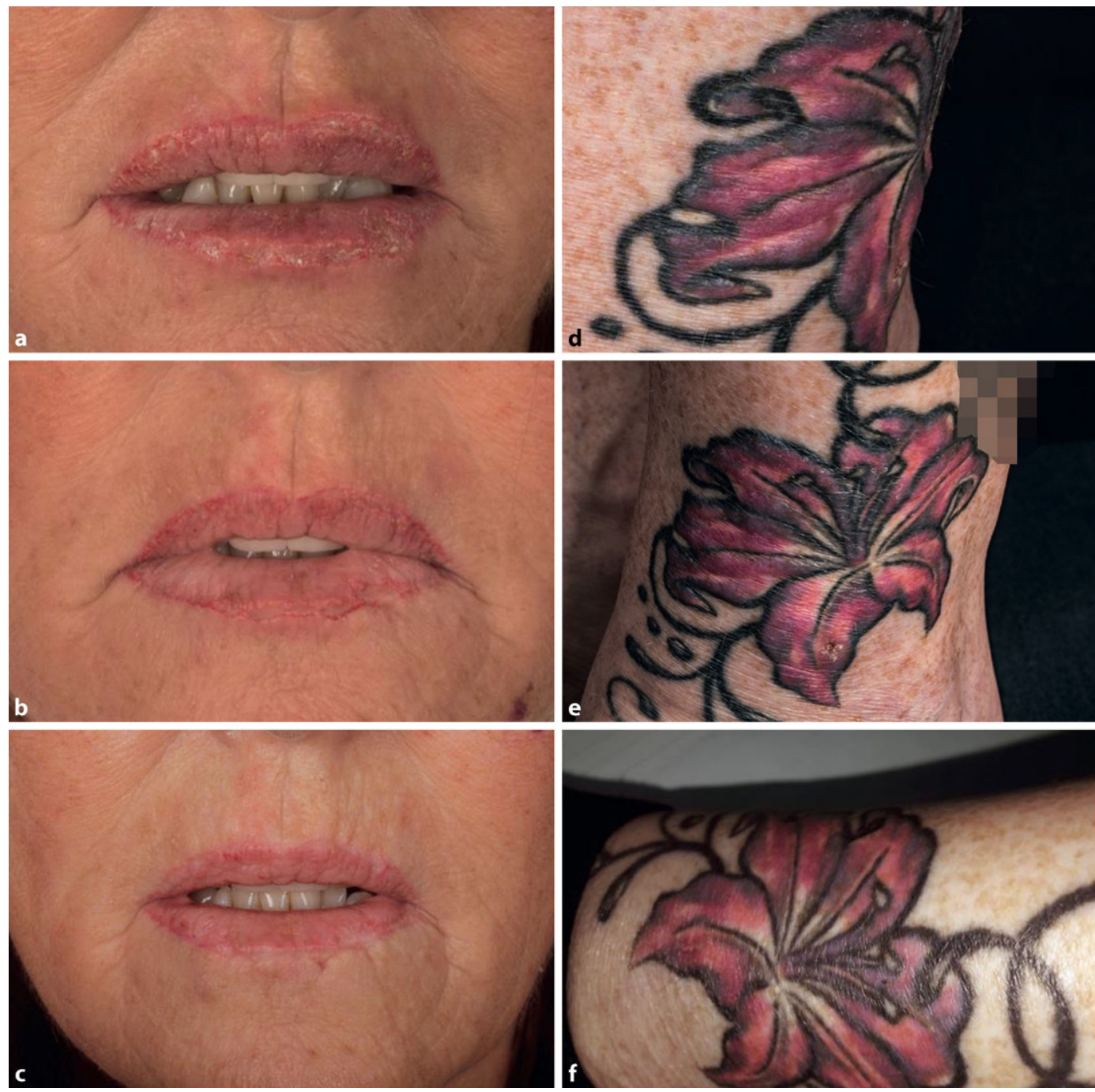

Abb. $1 \triangleleft$ Klinik. Fall 1:

Krustige und narbige Veränderungen im Bereich der Ober- und Unterlippe bei Erstvorstellung (a). Rückbildung der Knoten 7 Wochen (b) sowie knapp 2,5 Jahre (c) nach kortikosteroidhaltiger Injektion. Fall 2: Multiple Knoten im roten Bereich des Tattoos am linken Unterschenkel bei Erstvorstellung $(\mathbf{d}, \mathbf{e})$. Unauffälliges Tattoo 4 Wochen nach Behandlung (f) 
Fall 2. Histologisch fanden sich von einer Fibrose umgebene knotige Ansammlungen von Lymphozyten, Histiozyten und Plasmazellen. Zudem war im Corium feingranuläres, rotes, intra- und extrazelluläres Pigment erkennbar (• Abb. 2c, d).

\section{Diagnose}

Granulomatöse Entzündungsreaktion nach Permanent-Make-up bzw. Tätowierung mit rotem Farbstoff.

\section{Therapie und Verlauf}

Fall 1. Der Patientin wurde Mometasonfuroat-Creme 2-mal täglich über 2 Wochen an den betroffenen Hautarealen verordnet. Bei nur mäßigem Therapieansprechen erhielt die Patientin eine intraläsionale Probeinjektion mit Triamcinolonacetonid $40 \mathrm{mg}$ und Scandicain (Aspen Pharma Trading Limited, Dublin, Irland) $1 \%$ im Verhältnis 1:2 in den unteren linken Quadranten der Lippe. Aufgrund einer Befundbesserung im behandelten Bereich erfolgte 16 Tage später eine Injektion der Ober- und Unterlippe. Sieben Wochen nach der einmaligen Injektion war die gewohnte Lippenform zu verzeichnen (• Abb. 1b); 28 Monate später zeigte sich der Befund weiterhin unauffällig (• Abb. 1c).

Fall 2. Auch hier wurde wegen fehlenden Therapieansprechens auf Mometasonfuroat-Creme (2-mal täglich über 4 Wochen) eine Injektion nach obigem Schema im betroffenen Areal am linken Unterschenkel durchgeführt. Bereits nach 5 Wochen war kein knotiger Anteil mehr sichtbar (@ Abb. 1f). Die Patientin berichtete auch 10 Monate nach erfolgter Injektion über einen stabilen Befund.

\section{Diskussion}

In den letzten Jahren hat die Inzidenz von Tätowierungen stetig zugenommen $[1,10]$. Die Prävalenz liegt mittlerweile bei 3-8\% der Weltbevölkerung [6]. Das Permanent-Make-up stellt eine besondere Form der Tätowierung dar, bei der die Augenbrauen oder die Lippenkontur nachgezeichnet werden [2,3]. Infolge steigender Beliebtheit von Tattoos sind Dermatologen zunehmend mit unerwünschten Hautreaktionen nach Einbringen verschiedener Farbstoffe in die Haut konfrontiert [7, 8]. Komplikationen treten mit unterschiedlicher Latenz bis mehrere Jahre nach Applikation des Tattoos auf $[1,5,8,10]$. Neben viralen oder bakteriellen Infektionen wurde auch ein erhöhtes Aufkommen kutaner Tumoren berichtet $[4,9,10]$, wie beispielsweise Plattenepithel- oder Basalzellkarzinome [9]. Darüber hinaus können akute und chronische Entzündungen sowie allergische Reaktionen auf Inhaltsstoffe eines Tattoos auftreten [1]. Auch sind nichtinfektiöse, granulomatöse inflammatorische Reaktionen nach Tätowierungen beschrieben [1], für die eine Fremdkörperreaktion auf Pigment als ursächlich angenommen wird [6, 9]. Granulomatöse Reaktion treten insgesamt selten, am wahrscheinlichsten jedoch nach Einbringen von rotem und schwarzem Pigment auf $[1,4,5,8]$. Sarkoidale Fremdkörperreaktionen finden sich sogar häufiger nach schwarzen Tätowierungen. Wegen oftmals bestehender Unkenntnis über die genauen Inhaltsstoffe führt eine allergische Testung selten zu aussagekräftigen Ergebnissen [10]. Vereinzelt gibt es Berichte über eine fast vollständige Rückbildung der entzündlichen Reaktion nach Tätowierung durch eine anschließende topische oder intraläsionale Lokaltherapie mit Kortikosteroiden [2, 6]. Eine Exzision stellt eine alternative Therapieoption dar [2]. Jones et al. [3] berichten über eine Totalremission einer granulomatösen Entzündungsreaktion an der Lippe innerhalb von 4 Wochen nach erfolgter Biopsie. In den vorliegenden Fällen erwiesen sich die angewandten intraläsionalen kortikosteroidhaltigen Injektionen bei granulomatöser Reaktion als erfolgreich und komplikationslos.

\section{Fazit für die Praxis}

- Tätowierungen können unterschiedliche Komplikationen wie Infektionen oder entzündliche sowie allergische Reaktionen nach sich ziehen.

- Insbesondere nach Tätowierungen mit roten Farbstoffen treten Entzündungsreaktionen auf.
Hautarzt 2020 $\cdot 71: 805-808$

https://doi.org/10.1007/s00105-020-04654-8

(c) Der/die Autor(en) 2020

C. S. Cussigh - F. Toberer - A. Enk ·

H. A. Haenssle · C. Fink

Therapieansprechen auf intraläsionale

Steroidinjektionen bei granulomatöser Entzündungsreaktion nach Tätowierungen

\section{Zusammenfassung}

Tätowierungen inklusive PermanentMake-up können diverse Komplikationen wie virale oder bakterielle Infektionen sowie allergische und entzündliche Reaktionen nach sich ziehen. Bei Letzteren weist die Histologie neben exogenem Pigment ein Entzündungsinfiltrat auf, das je nach Reaktionsmuster lymphozytär oder histiozytär-granulomatös dominiert sein kann. Nachfolgend wird über die erfolgreiche Therapie mittels intraläsionaler Triamcinolonacetonid-Injektionen bei granulomatöser Entzündungsreaktion nach Tätowierungen in 2 Fällen berichtet.

Schlüsselwörter

Permanent-Make-up · Granulom . Infektionen - Allergische Reaktionen .

Triamcinolonacetonid-Injektionen

Effectiveness of intralesional steroid injections in granulomatous inflammation after tattooing

\section{Abstract}

Tattoos, including permanent makeup, may entail diverse complications like viral or bacterial infections and allergic and inflammatory reactions. In the latter case, besides exogenous pigment, histology shows an either lymphocytic or histiocyticgranulomatous infiltrate, depending on the predominant reaction pattern. We report successful treatment with intralesional triamcinolone acetonide injections in two individuals who developed granulomatous inflammation after tattooing.

Keywords

Permanent makeup - Granuloma - Infections . Allergic reactions - Triamcinolone acetonide injections 


\section{Kasuistiken}
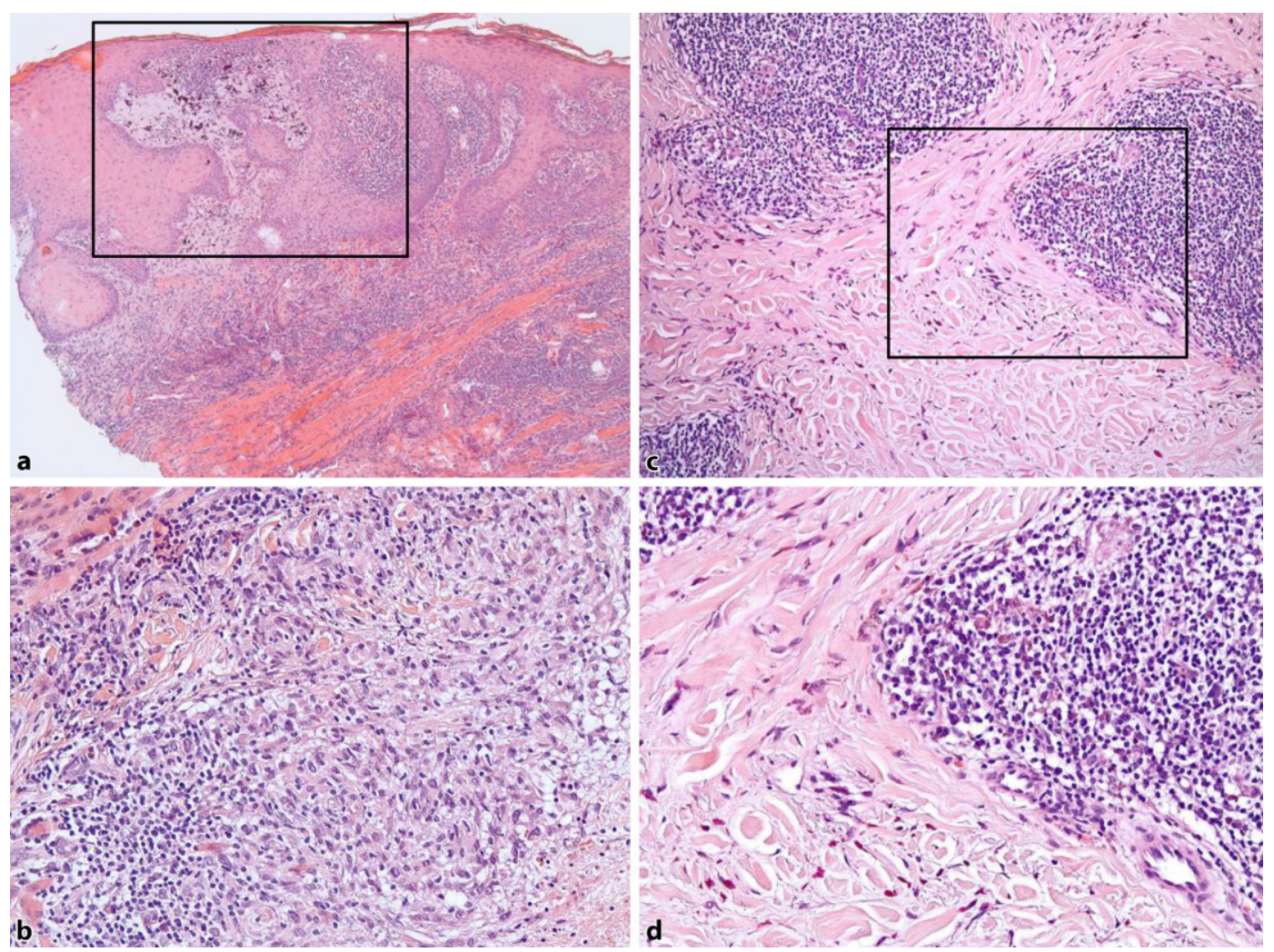

Abb. $2 \triangleleft$ Histologischer Befund. Fall 1: Die Histologie zeigt ein hyperplastisches Epithel sowie rote und schwarze Pigmenteinschlüsse in der oberen Dermis. Zudem findet sich ein lymphozytäres Infiltrat mit Plasmazellen (a HE[Hämatoxylin-Eosin]Färbung, Originalvergr. 50:1). b Darstellung des Infiltrats, bestehend auf Plasmazellen und Lymphozyten, bei 200-facher Vergrößerung. Fall 2: Histologisch lassen sich im Korium knotig imponierende Infiltrate aus Lymphozyten, Histiozyten und Plasmazellen erkennen, die von einem fibrosierten Bindegewebe umgeben sind. Teils intrazellulär, teils extrazellulär gelegenes rotes, feingranuläres Pigment (c HE-Färbung, Originalvergr. 50:1). Der Auswahlkasten in c wird in $\mathbf{d}$ bei 100 -facher Vergrößerung dargestellt

\section{- Intraläsionale kortikosteroidhaltige Injektionen stellen eine effektive Therapieoption dar.}

\section{Korrespondenzadresse}

\section{Dr. med. Christine Fink, MD}

Klinik für Dermatologie, Venerologie und Allergologie, Universitätsklinikum Heidelberg (UKH), Ruprecht-Karls-Universität Im Neuenheimer Feld 440, 69120 Heidelberg Deutschland christine.fink@med.uni-heidelberg.de

Funding. Open Access funding provided by Projekt DEAL.

\section{Einhaltung ethischer Richtlinien}

Interessenkonflikt. C.S. Cussigh, F. Toberer, A. Enk H.A. Haenssle und C. Fink geben an, dass kein Interessenkonflikt besteht.

Dieser Beitrag beinhaltet keine von den Autoren durchgeführten Studien an Menschen oder Tieren. Alle Patienten, die über Bildmaterial oder anderweitige Angaben innerhalb des Manuskripts zu identifizieren sind, haben hierzu ihre schriftliche Einwilligung gegeben.

Open Access. Dieser Artikel wird unter der Creative Commons Namensnennung 4.0 International Lizenz veröffentlicht, welche die Nutzung, Vervielfältigung, Bearbeitung, Verbreitung und Wiedergabe in jeglichem Medium und Format erlaubt, sofern Sie den/die ursprünglichen Autor(en) und die Quelle ordnungsgemäß nennen, einen Link zur Creative Commons Lizenz beifügen und angeben, ob Änderungen vorgenommen wurden.

Die in diesem Artikel enthaltenen Bilder und sonstiges Drittmaterial unterliegen ebenfalls der genannten Creative Commons Lizenz, sofern sich aus der Abbildungslegende nichts anderes ergibt. Sofern das betreffende Material nicht unter der genannten Creative Commons Lizenz steht und die betreffende Handlung nicht nach gesetzlichen Vorschriften erlaubt ist, ist für die oben aufgeführten Weiterverwendungen des $\mathrm{Ma}$ terials die Einwilligung des jeweiligen Rechteinhabers einzuholen.

Weitere Details zur Lizenz entnehmen Sie bitte der Lizenzinformation auf http://creativecommons.org/ licenses/by/4.0/deed.de.

\section{Literatur}

1. González-Villanueva I, Silvestre Salvador J (2018) ¿Qué procedimientos diagnósticos deberíamos realizarante una sospecha de reacción alérgicaaun tatuaje? Propuesta basada en nuestra casuística. Actas Dermosifiliogr 109:162-172

2. Huisman $S$, van der Bent SAS, Wolkerstorfer $A$ Rustemeyer T (2019) Granulomatous tattoo reactions in permanent makeup of the eyebrows. J Cosmet Dermatol 18:212-214

3. Jones B, Oh C, Egan CA (2008) Spontaneous resolution of a delayed granulomatous reaction to cosmetic tattoo. Int J Dermatol 47:59-60
4. Kluger N (2015) Tattooed people, who are you? Demographic and behavioral characteristics of tattooed individuals. Ann Dermatol Venereol 142(6/7):410-420

5. Mortimer NJ, Chave TA, Johnston GA (2003) Red tattoo reactions. Clin Exp Dermatol 28:508-510

6. Quint KD, Genders RE, Vermeer MH (2012) A delayed granulomatous reaction to a cosmetic tattoo of the eyebrows: a report of total regression after intralesional corticosteroid injections. Dermatol Surg 38:951-953

7. Serup J, Sepehri M, Hutton Carlsen K (2016) Classification of tattoo complications in a hospital material of 493 adverse events. Dermatology 232:668-678

8. Shinohara MM (2016) Complications of decorative tattoo. Clin Dermatol 34:287-292

9. Sweeney SA, Hicks LD, Ranallo N, Snyder N 4th, Soldano AC (2013) Perforating granulomatous dermatitis reaction to exogenous tattoo pigment: a case report and review of the literature. Am J Dermatopathol 35:754-756

10. Wenzel SM, Rittmann I, Landthaler M, Bäumler W (2013) Adverse reactions after tattooing: review of the literature and comparison to results of a survey. Dermatology 22:138-147 